\title{
Persistence of risk of death after hospital discharge to locations other than home after cardiac surgery
}

Makoto Mori, MD, ${ }^{\mathrm{a}}$ Syed Usman Bin Mahmood, MBBS, ${ }^{\mathrm{a}}$ Haoran Zhuo, MPH, ${ }^{\mathrm{b}}$ Sameh Yousef, MD, ${ }^{\mathrm{a}}$ Jeremy Green, BA, ${ }^{\mathrm{c}}$ Abeel A. Mangi, MD, ${ }^{\mathrm{a}}$ Yawei Zhang, MD, PhD, MPH, ${ }^{\mathrm{b}}$ and Arnar Geirsson, $\mathrm{MD}^{\mathrm{a}}$

\section{ABSTRACT}

Objective: Temporal changes in the risk of postoperative death following cardiac surgery are uncharacterized. We aimed to quantify the duration of postoperative phase with elevated risk of death in patients who underwent cardiac surgery and were discharged to home and destinations other than home.

Method: We conducted a retrospective cohort study of 6894 patients who underwent cardiac surgery between 2008 and 2016 at a tertiary care center in the United States. Logistic regression models with restricted cubic splining along the days since hospital discharge were fitted for risk of death in patients who were discharged to home and those discharged to destinations other than home. The splining curves were analyzed to quantify the duration of postoperative high-risk phase in each cohort.

Results: Mortality rate was significantly higher in the nonhome cohort compared with those discharged to home at 365 days following hospital discharge $(9.3 \%$ vs $2.1 \% ; P<.001)$. Discharge to destinations other than home was an independent predictor of late death (hazard ratio, 1.36; $95 \%$ confidence interval, 1.15-1.61; $P<.001)$. Analysis of restricted cubic splining curves demonstrated that the postoperative phase with elevated risk of death persisted for 80.3 days in nonhome discharge cohorts, whereas a comparable phase was nonexistent in patients who went home. Predictors of nonhome discharge were identified, with combination of preoperative and postoperative variables yielding $C$ statistics of 0.83 .

Conclusions: Hospital discharges to locations other than home following cardiac surgery were associated with an increased risk of late mortality. The postoperative high-risk phase persisted for 80 days in patients who were discharged to locations other than home, supporting the use of 90-day outcome measures as quality metrics. Predictors of discharge to locations other than home were identified, and this may aid in selective intervention to reduce the risk of death in this vulnerable patient population. (J Thorac Cardiovasc Surg 2020;159:528-35)

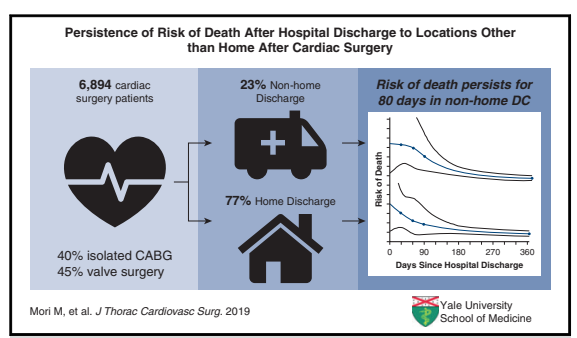

Persistent high-risk phase up to 80 days following cardiac surgery in patients discharged to locations other than home.2

\section{Central Message}

Following cardiac surgery, the postoperative high-risk phase persists up to 80 days in patients discharged to destinations other than home, whereas this phase is nonexistent in patients who are discharged home.

\section{Perspective}

The duration of postoperative phase at which a patient continues to be at high risk for mortality is unknown. This study identified that following cardiac surgery, patients who were discharged to locations other than home experienced elevated risk of death up to 80 days following the operation. This phase was not observed in patients who went home directly.

See Commentaries on pages 536 and 538.
From the ${ }^{\mathrm{a} S e c t i o n}$ of Cardiac Surgery, School of Medicine, and ${ }^{\mathrm{b}}$ Section of Surgical Outcomes and Epidemiology, School of Public Health, Yale University, New Haven, Conn; and ${ }^{\mathrm{c}}$ Frank H. Netter School of Medicine, Quinnipiac University, Hamden, Conn.

Supported by the Yale Clinical and Translational Science Award, grant UL1TR001863, from the National Center for Advancing Translational Science, a component of the National Institutes of Health.

Read at the 99th Annual Meeting of The American Association for Thoracic Surgery, Toronto, Ontario, Canada, May 4-7, 2019.

Received for publication Aug 21, 2018; revisions received Feb 11, 2019; accepted for publication Feb 18, 2019; available ahead of print July 2, 2019.

Address for reprints: Arnar Geirsson, MD, Section of Cardiac Surgery, School of Medicine, Yale University, PO Box 208039, New Haven, CT 06510 (E-mail: arnar.geirsson@yale.edu).

$0022-5223 / \$ 36.00$

Copyright @ 2019 Published by Elsevier Inc. on behalf of The American Association for Thoracic Surgery

https://doi.org/10.1016/j.jtcvs.2019.02.079
Thirty-day mortality measure is a commonly used metric to assess outcomes of various surgical approaches and overall quality of patient care. National and state-level data illustrate that events occurring beyond 30 days following index cardiac operations are substantial, ${ }^{1,2}$ suggesting that a longer timeframe beyond 30 days would serve as a more

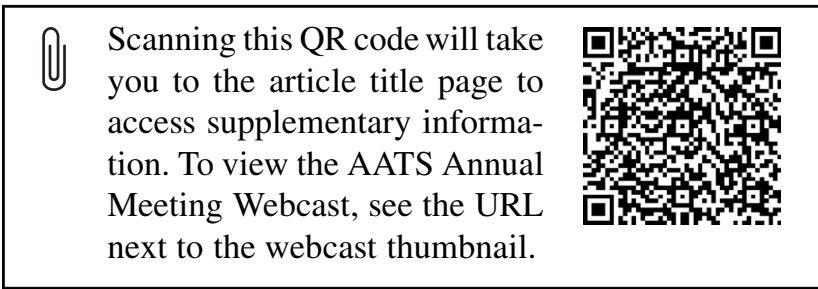



Abbreviations and Acronyms
AUC = area under the curve
$\mathrm{CABG}=$ coronary artery bypass graft
ICU = intensive care unit
RCS = restricted cubic splining
STS $=$ Society of Thoracic Surgeons

robust metric to assess surgical outcomes. For instance, readmission following coronary artery bypass graft surgery (CABG) has been shown to be associated with an increased risk of 90-day mortality and is associated with considerable increase in the cost of the index operation., The common ground in the efforts characterizing postoperative outcomes beyond 30 days is the notion that the mortality risk of surgery persists beyond 30 days, and that the longer time frame is more appropriate in capturing events attributable to undergoing surgery. The risk of death following surgery is dynamic and is expected to diminish as longer time lapses from the surgery. However, changes in the risk of death along time following cardiac surgery has not been well characterized, and the duration during which patients who underwent surgery continues to be at increase risk of death remains unknown.

Status of hospital discharges to destinations other than home may serve as a meaningful marker of patients who harbor persistently increased risk of mortality following hospital discharge, as the status of nonhome discharge likely encompasses patient comorbidity, postoperative complications, and the comprehensive assessment of patients' trajectory of recovery by the providers. Several studies have evaluated predictors of nonhome discharge and midterm outcomes following cardiac surgery. 5,6 Based on existing studies and general observation that patients who cannot be discharged to home comprise a sicker population, worse outcome in this group is expected. However, the dynamic nature of the postdischarge risk of death has not been characterized in this setting. In this study, we aimed to characterize late outcomes in patients who were discharged to home and to locations other than home following cardiac surgery, and quantify dynamic changes in the risk of mortality along time since discharge. We also aimed to identify predictors of nonhome discharge to evaluate whether preoperative identification of such patient group would be possible.

\section{METHODS \\ Patient Population}

We conducted a single-institution, retrospective review of 7067 consecutive patients undergoing coronary artery bypass surgery or valve surgeries between 2008 and 2016 at a tertiary care hospital in the United States. Because the state vital statistics record only captures patients with Connecticut residence, out-of-state patients were excluded from the analysis.
TABLE 1. Baseline characteristics

\begin{tabular}{|c|c|c|c|}
\hline Variable & $\begin{array}{l}\text { Nonhome } \\
\text { discharge }\end{array}$ & $\begin{array}{c}\text { Home } \\
\text { discharge }\end{array}$ & $P$ value* \\
\hline Sample size & 1595 & 5299 & \\
\hline Age (y) & $73.0 \pm 11.9$ & $64.7 \pm 12.4$ & $<.0001$ \\
\hline Female & $701(43.9)$ & $1502(28.3)$ & $<.0001$ \\
\hline Race & & & $<.0001$ \\
\hline White & $1406(88.2)$ & $4729(89.2)$ & \\
\hline African American & $122(7.6)$ & $239(4.5)$ & \\
\hline Asian & $7(0.4)$ & $89(1.7)$ & \\
\hline Others & $60(3.8)$ & $242(4.6)$ & \\
\hline Body mass index $\dagger$ & $28.1(24.4-32.7)$ & $28.1(25.0-32.0)$ & .68 \\
\hline Diabetes & & & $<.0001$ \\
\hline Noninsulin-dependent & $372(23.3)$ & $1078(20.3)$ & \\
\hline Insulin-dependent & $256(16.1)$ & $431(8.1)$ & \\
\hline Dyslipidemia & $1225(76.8)$ & $3862(72.9)$ & .0018 \\
\hline $\begin{array}{l}\text { eGFR } \\
\quad\left(\mathrm{mL} / \mathrm{min} / 1.73 \mathrm{~m}^{2}\right) \dagger\end{array}$ & $71.0(51.5-92.5)$ & $79.4(64.7-95.8)$ & $<.0001$ \\
\hline Preoperative dialysis & $79(5.0)$ & $82(1.5)$ & $<.0001$ \\
\hline Hypertension & $1379(86.5)$ & $4169(78.7)$ & $<.0001$ \\
\hline History of endocarditis & $138(8.7)$ & $173(3.3)$ & $<.0001$ \\
\hline Chronic lung disease & $135(8.5)$ & $238(4.5)$ & $<.0001$ \\
\hline Immunosuppressed & $102(6.4)$ & 207 (3.9) & $<.0001$ \\
\hline PVD & $316(19.8)$ & $680(12.8)$ & $<.0001$ \\
\hline History of stroke & $244(15.3)$ & $297(5.6)$ & $<.0001$ \\
\hline Prior MI & $678(42.5)$ & $1591(30.0)$ & $<.0001$ \\
\hline Cardiogenic shock & $71(4.5)$ & $72(1.4)$ & $<.0001$ \\
\hline Redo sternotomy & $145(9.1)$ & $420(7.9)$ & .13 \\
\hline Congestive heart failure & $751(47.1)$ & $1220(23.0)$ & $<.0001$ \\
\hline Ejection fraction $(\%) \dagger$ & $56(43-64)$ & $60(50-65)$ & $<.0001$ \\
\hline Status of the procedure & & & $<.0001$ \\
\hline Elective & $731(45.8)$ & $3576(67.5)$ & \\
\hline Urgent & $768(48.2)$ & $1589(30.0)$ & \\
\hline Emergent & $92(5.8)$ & $134(2.5)$ & \\
\hline
\end{tabular}

Values are presented as $\mathrm{n}$, mean \pm standard deviation, or median (interquartile range). $e G F R$, Estimated glomerular filtration rate; $P V D$, peripheral vascular disease; $M I$, myocardial infarction. *Statistical significance testing was performed with nonparametric testing (Wilcoxon rank-sum test). †Nonnormal distribution of continuous variables.

Patients were initially categorized by the discharge location (ie, nursing home, extended care/transition care rehabilitation facility, other hospital, hospice, and home), and this was then dichotomized into home or places other than home. Patients who died before hospital discharge were excluded $(\mathrm{n}=169)$. Patients who were discharged to hospice were excluded $(n=4)$ because the incidence was rare and this population was expected to portend poor prognosis that deviates significantly from that of rest of the cohort. The final cohort included in the analysis consisted of 6894 patients. The Institutional Review Board at Yale University approved this study and individual consent was waived.

\section{Collected Data and Outcomes}

Baseline demographic and comorbidity data, outlined in Table 1, and operative and postoperative data, outlined in Table 2, were collected. Patient medical record was abstracted into the Society of Thoracic Surgeons (STS) database, and this database was queried for the analysis. The STS data definitions (versions 2.61, 2.81, and 2.73) were used (https://www. 
TABLE 2. Case type and postoperative complications

\begin{tabular}{lccc}
\hline \multicolumn{1}{c}{ Variable } & $\begin{array}{c}\text { Nonhome } \\
\text { discharge }\end{array}$ & $\begin{array}{c}\text { Home } \\
\text { discharge }\end{array}$ & $\boldsymbol{P}$ value* \\
\hline Case type & & & $<.0001$ \\
$\quad$ Isolated CABG & $611(38.3)$ & $2210(41.1)$ & \\
Isolated single valve & $575(36.1)$ & $2188(41.3)$ & \\
Isolated multiple valve & $114(7.1)$ & $215(4.1)$ & \\
CABG + single valve & $254(15.9)$ & $611(11.5)$ & \\
CABG + multiple valve & $22(1.4)$ & $41(0.8)$ & \\
Postoperative variables & & & \\
Reoperation for bleeding & $70(4.4)$ & $108(2.0)$ & $<.0001$ \\
Stroke & $71(4.5)$ & $25(0.5)$ & $<.0001$ \\
Prolonged ventilation & $419(26.3)$ & $420(7.9)$ & $<.0001$ \\
Pneumonia & $203(12.7)$ & $142(2.7)$ & $<.0001$ \\
Renal failure & $49(3.1)$ & $51(1.0)$ & $<.0001$ \\
Renal failure with dialysis & $14(0.9)$ & $8(0.2)$ & $<.0001$ \\
ICU Length of stay $(\mathrm{d}) \dagger$ & $4.0(2.8-6.7)$ & $2.3(1.9-3.8)$ & $<.0001$ \\
\hline
\end{tabular}

Values are presented as $\mathrm{n}(\%)$ or median (interquartile range). $C A B G$, Coronary artery bypass grafting; $I C U$, intensive care unit. *Statistical significance testing was performed with nonparametric testing (Wilcoxon rank-sum test). †Indicates nonnormal distribution of continuous variables.

sts.org). Preoperative stroke was defined as those occurring within 30 days of surgery. Estimated glomerular filtration rate was calculated using Modification of Diet in Renal Disease equation ${ }^{7}$ based on the last available creatinine values before the surgery.

The evaluated outcomes were survival at $30,60,90$, and 365 days from the time of hospital discharge. Time of hospital discharge was used as time 0 , instead of time of surgery, because this analysis excluded patients who died during the index hospitalization. Long-term survival was evaluated up to 5 years, although 5-year follow-up data was incomplete due to a proportion of patients undergoing surgery within 5 years of the last follow-up date. The detail is described in the result section. Postdischarge survival was ascertained by linking the institutional patient-level data to Connecticut State Department of Public Health death records, which capture all mortality that occurred in Connecticut residents. The final date of follow-up was December 31, 2016.

Following STS Adult Cardiac Surgery Database definitions were used for the postoperative complications: postoperative prolonged intubation as mechanical ventilation $>24$ hours, postoperative renal failure as either new dialysis requirement in the postoperative period, increase in serum creatinine level $3 \times$ greater than baseline, or postoperative serum creatinine level $\geq 4 \mathrm{mg} / \mathrm{dL}$, and stroke as neurologic deficit of abrupt onset caused by a disturbance in blood supply to the brain that did not resolve within 24 hours. Postoperative complications were collected up to the time of hospital discharge.

Missing data were handled in accordance to the method described by Shahian and colleagues ${ }^{8}$ in the 2008 STS risk model development. Briefly, the method involves categorizing missing risk variables into the lowest risk category, conditioning missing ejection fraction to sex and congestive heart failure diagnosis, and conditioning body surface area to sex.

\section{Statistical Analysis}

Differences in the patient characteristics between patients discharged to home and those discharged to places other than home were compared with 2 -tailed $t$ test for continuous variables, $\chi^{2}$ test for categorical variables, or Fisher exact test for categorical variables with cell count $\leq 5$. For continuous variables violating normality assumptions, Wilcoxon rank-sum test was used for bivariate comparisons with reporting of median and interquartile range (IQR). Continuous variables were summarized by mean with standard deviation, unless otherwise specified. Categorical variables are summarized by percentages. Assuming rare incidences of the primary outcome (ie, death), odds ratios derived from models are assumed to approximate relative risk and referred to as risk of death.

Kaplan-Meier analysis was used to estimate long-term survival from the time of hospital discharge. Log-rank test was used to assess the long-term survival difference between patients with home discharge and nonhome discharge. Cox proportional hazard model was fitted to the entire dataset to assess adjusted risk of nonhome discharge associated with long-term survival, using the preoperative, case type, and postoperative variables as input covariates (Table E1). The proportional hazards assumption in the Cox model for covariates was tested by examining the log-log Kaplan-Meier curves of the covariates, and variables violating the assumption were stratified in the final Cox model. To evaluate the adjusted risk of death along the time from hospital discharge in the cohorts of home discharge and nonhome discharge, logistic regression restricted cubic splining (RCS $)^{10}$ was performed along the continuum of days from hospital discharge with 4 knots at 30,60, 90, and 365 days, with input variables consisting of preoperative, case type, and postoperative variables. RCS was performed separately on dataset only consisting of patients with home discharge, and dataset only consisting of patients with nonhome discharge, with the intention of evaluating the differences in temporal persistence of mortality risk between home and nonhome discharge cohorts.

To identify predictors of nonhome discharge, 4 logistic regression models with forward selection and backward elimination were fitted: preoperative variables only, preoperative and case type variables, postoperative variables only, and preoperative and postoperative variables. Sets of variables included in the model are delineated in Table E1. Discriminatory ability of the models were evaluated via receiver-operator characteristics analysis with the comparison of $C$ index.

A $P$ value $<.05$ was used to define statistically significant differences and correlations. For multiple models sharing same covariates, Bonferroni correction was applied yield lower $P$-value threshold for statistical significance. Variables below the corrected $P$-value threshold are presented in boldface type in Table 3 and 4. All analysis was conducted with SAS version 9.4 (SAS Institute Inc, Cary, NC).

\section{RESULTS}

Baseline patient characteristics and comorbidity are outlined in Table 1. Compared with patients who were discharged to home following cardiac surgery, patients who were discharged to locations other than home were older $(73.0 \pm 11.9$ years vs $64.7 \pm 12.4$ years; $P<.001)$ and harbored comorbidities more frequently. The proportions of nonelective cases were significantly higher in patients discharged to location other than home $(48.2 \%$ vs $30.0 \%$ for urgent and $5.8 \%$ vs $2.5 \%$ for emergent cases $[P<.001])$. Table 2 outlines the distribution of case types, postoperative complications, and intensive care unit (ICU) length of stay. The distribution of case types was similar, but higher proportions of nonhome discharge patients underwent multivalve and concomitant $\mathrm{CABG}$ plus valve procedures. All evaluated complications were statistically significantly more common and ICU stay was longer in the nonhome discharge cohort $(6.0 \pm 7.3$ days vs 3.0 vs 2.0 days; $P<.001)$. Unadjusted mortality rates were significantly higher in nonhome cohort compared with those discharged to home at all of the following time points from the time of hospital discharge: 30 days $(0.8 \%$ vs $0.2 \%$; $P<.001), 60$ days $(2.4 \%$ vs $0.4 \% ; P<.001), 90$ days 
TABLE 3. Independent risk factors of death following hospital discharge

\begin{tabular}{lcc}
\hline \multicolumn{1}{c}{ Variables } & $\begin{array}{c}\text { Hazard ratio (95\% } \\
\text { confidence interval) }\end{array}$ & $\boldsymbol{P}$ value* \\
\hline Nonhome destination & $1.36(1.15-1.61)$ & $\mathbf{. 0 0 0 3}$ \\
\hline Age $\dagger$ & $1.06(1.05-1.06)$ & $<.000$ \\
\hline Female sex & $1.32(1.13-1.55)$ & $\mathbf{. 0 0 0 6}$ \\
\hline Nonwhite race & $1.39(1.05-1.82)$ & $\mathbf{. 0 1 9}$ \\
\hline Diabetes & $1.28(1.07-1.53)$ & $\mathbf{. 0 0 8 0}$ \\
\hline eGFR $†$ & $1.00(0.99-1.00)$ & $\mathbf{. 0 0 3 5}$ \\
\hline Infective endocarditis & $1.22(1.02-1.46)$ & .028 \\
\hline Preoperative dialysis & $1.78(1.27-2.48)$ & $\mathbf{. 0 0 0 7}$ \\
\hline Chronic lung disease & $1.74(1.40-2.16)$ & $<.0001$ \\
\hline Immunosuppressed & $2.01(1.57-2.58)$ & $<. \mathbf{0 0 0 1}$ \\
\hline PVD & $1.46(1.23-1.73)$ & $<.0001$ \\
\hline CHF & $1.47(1.24-1.73)$ & $<.0001$ \\
\hline Ejection fraction $\S$ & $0.99(0.99-1.00)$ & $\mathbf{. 0 0 9}$ \\
\hline Redo sternotomy & $1.53(1.13-2.08)$ & $\mathbf{. 0 0 6 2}$ \\
\hline Prolonged ventilation & $1.48(1.23-1.77)$ & $<.0001$ \\
\hline ICU LOS $\|$ & $1.02(1.01-1.03)$ & $\mathbf{. 0 0 0 4}$ \\
\hline
\end{tabular}

eGFR, Estimated glomerular filtration rate; $P V D$, peripheral vascular disease; $C H F$, congestive heart failure; ICU, intensive care unit; LOS, length of stay. *Boldface type signifies statistical significance after Bonferroni correction. $\nmid$ Per 1 year. $\ddagger$ Per unit increase. §Per percent. $\|$ Per 1 day.

(3.2\% vs $0.6 \% ; P<.001)$, and 365 days $(9.3 \%$ vs $2.1 \%$; $P<.001)$. Median duration of follow-up was 34.2 months (IQR, 14.7-54.7 months) for nonhome cohort and 45.0 months (IQR, 22.4-75.8 months) for the home discharge cohort.

Kaplan-Meier curve by destinations after hospital discharge is shown in Figure 1. Patients discharged to locations other than home had significantly worse survival compared with those discharged to home $(P<.001$ by log-rank).

Independent predictors of death following hospital discharge, evaluated via Cox proportional hazard model with the input variables listed in Table E1, are summarized in Table 3. Discharge to destinations other than home was associated with an increased risk of death (hazard ratio, $1.36 ; 95 \%$ confidence interval, $1.15-1.61 ; P<.001)$. Strong predictors of death included immunosuppressed status, peripheral vascular disease, congestive heart failure, redo sternotomy, and prolonged need for mechanical ventilator support.

RCS was performed to evaluate changes in the risk of death following hospital discharge along the continuum of days following hospital discharge (Figure 2). RCS demonstrated that the risk of death continues to persist in both home and nonhome discharge cohorts, but persisted much longer in the nonhome cohort. Although the risk of death immediately begins to decline in home discharge cohort,
TABLE 4. Predictors of disposition to locations other than home

\begin{tabular}{|c|c|c|c|c|}
\hline \multirow[b]{2}{*}{ Variable } & \multirow{2}{*}{$\begin{array}{l}\text { Odds } \\
\text { ratio }\end{array}$} & \multicolumn{2}{|c|}{$\begin{array}{l}95 \% \text { confidence } \\
\text { interval }\end{array}$} & \multirow[b]{2}{*}{$P$ value* } \\
\hline & & $2.5 \%$ & $97.5 \%$ & \\
\hline \multicolumn{5}{|l|}{ Preoperative variables } \\
\hline Age $\dagger$ & 1.07 & 1.06 & 1.08 & $<.0001$ \\
\hline Female $\ddagger$ & 1.66 & 1.44 & 1.91 & $<.0001$ \\
\hline \multicolumn{5}{|l|}{ Race $\S$} \\
\hline African American & 1.66 & 1.25 & 2.21 & .0005 \\
\hline Asian & 0.18 & 0.06 & 0.48 & .0007 \\
\hline Other & 0.89 & 0.63 & 1.25 & .51 \\
\hline Body mass index $\|$ & 1.02 & 1.01 & 1.03 & .0003 \\
\hline \multicolumn{5}{|l|}{ Diabetes $\llbracket$} \\
\hline Noninsulin dependent & 1.22 & 1.03 & 1.44 & .023 \\
\hline Insulin dependent & 1.89 & 1.53 & 2.34 & $<.0001$ \\
\hline Infective endocarditis & 2.54 & 2.11 & 3.06 & $<.0001$ \\
\hline Dialysis & 1.55 & 1.04 & 2.30 & .031 \\
\hline Lung disease & 1.34 & 1.03 & 1.74 & .027 \\
\hline Stroke & 2.20 & 1.77 & 2.74 & $<.0001$ \\
\hline Peripheral vascular disease & 1.22 & 1.02 & 1.46 & .029 \\
\hline Congestive heart failure & 1.62 & 1.40 & 1.87 & $<.0001$ \\
\hline \multicolumn{5}{|l|}{ Case status\# } \\
\hline Urgent & 1.95 & 1.69 & 2.24 & $<.0001$ \\
\hline mergent & 2.10 & 1.41 & 3.12 & .0003 \\
\hline Prior sternotomy & 0.63 & 0.49 & 0.81 & .0004 \\
\hline \multicolumn{5}{|l|}{ Complications } \\
\hline Stroke & 4.33 & 2.51 & 7.48 & $<.0001$ \\
\hline $\begin{array}{l}\text { Prolonged mechanical } \\
\text { ventilation }\end{array}$ & 1.30 & 1.05 & 1.60 & .017 \\
\hline Pneumonia & 1.57 & 1.14 & 2.15 & .005 \\
\hline ICU length of stay** & 1.18 & 1.15 & 1.21 & $<.0001$ \\
\hline
\end{tabular}

ICU, Intensive care unit. *Boldface type signifies statistical significance after Bonferroni correction. $\dagger$ Per unit increase. $\ddagger$ Male is reference category. $\S$ White is reference category. ||Per unit increase. $\uparrow$ No is reference category. \#Elective is reference category. **Per 1 day increase.

the risk of death has a plateau phase in nonhome discharge cohort that persists to about 80 days (Figure E1) following discharge, indicating that the nonhome discharge cohort is at increased risk of death for a longer period of time following hospital discharge compared with those discharged to home.

Predictors of nonhome discharge were evaluated with logistic regression models. Results of the models incorporating combination of preoperative, case type, and postoperative variables are demonstrated in Table E1 and Figure E1. Discriminatory ability of each model was assessed with receiver operator characteristics analysis and yielded the following $C$ statistics values: preoperative variables only (area under the curve [AUC], 0.796), preoperative variables plus case types (AUC, 0.794), postoperative variables only (AUC, 0.714), and preoperative variables plus postoperative variables (AUC, 0.828). In the model combining preoperative and postoperative variables, variables strongly associated with nonhome discharge included 


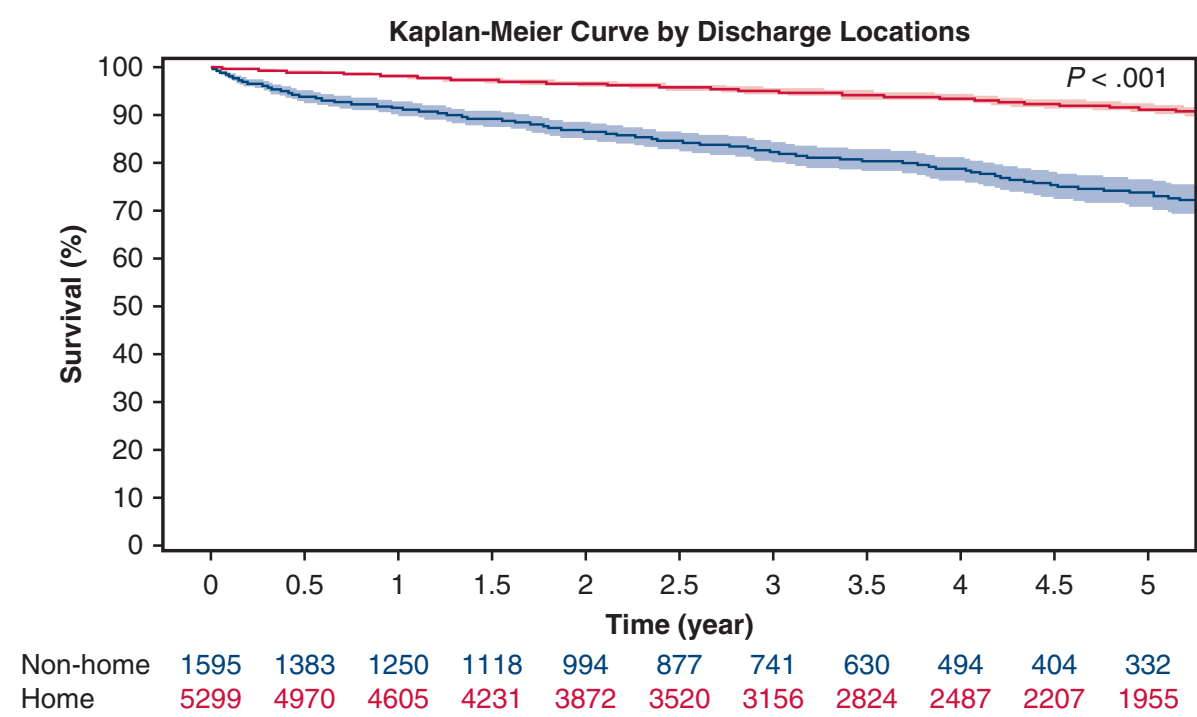

FIGURE 1. Kaplan-Meier curve by discharge locations. The figure shows long-term survival by discharge location: home (red) and places other than home (blue). The data are shown up to 5 years following hospital discharge. Log-rank test demonstrated statistically significant difference in survival $(P<.001)$.

female sex, African American race, infective endocarditis, history of stroke, emergent case status, postoperative stroke, ICU length of stay, and prolonged mechanical ventilator requirement.

\section{DISCUSSION}

The salient findings of this study are the following: patients who were discharged to locations other than home had a significantly higher risk of late mortality; the rate of decline in the risk of death following hospital discharge differed between those discharged to home and nonhome, with nonhome discharge cohort harboring persistently elevated risk of death until approximately 80 days following hospital discharge, after which the risk showed a temporal decline comparable to that of home discharge cohort; and nonhome discharge was associated most strongly with preoperative and postoperative factors than case types, with preoperative factors showing a strong discrimination, implicating that preoperative identification of this patient population is possible. This may aid in preoperative decision making and patient counseling, and may allow for selective intervention before the operation when possible.

Analysis of national STS Adult Cardiac Surgery Database linked with Medicare data demonstrated that the kinetics of mortality occurring up to 3 years after CABG differs by patient comorbidity, based on stratified KaplanMeier analysis. ${ }^{11}$ The focus on outcome measures beyond 30 days is being advocated by observational data suggesting that 30 days may be inadequate in capturing events relevant to index surgery. ${ }^{2}$ Our findings on the persistent risk of mortality in the subgroup of patients who were discharged to locations other than home is in line with this argument. Implementing this in a payment model was met with conflicting responses from stakeholders, resulting in the Centers for Medicare and Medicaid Services renouncing its original intent to implement CABG bundled payment model that encompasses 90 days after surgery as the payment period. Indeed, the issue requires considerations to the complexity introduced by a longer follow-up time, including the introduction of noise related to events that are unrelated to surgery or care. Further evaluation of long-term outcome measures will allow for an informed decision making with regard to whether to promote implementation of such measures.

The population of patients undergoing cardiac surgery is aging $^{12,13}$ and our study demonstrated that almost one quarter $(23 \%)$ of patients undergoing cardiac surgery are discharged to nonhome destinations with increasing age as a strong predictor of nonhome discharge. The incidence of nonhome discharge at a national scale in the United States is unknown, but a single-center study of 590 cardiac surgery patients reported $15.6 \%$ of patients being discharged to location other than home, ${ }^{5}$ a proportion comparable to our study. Although not directly comparable, data from Danish national registry reported a strikingly low incidence of $1.4 \%$ for nursing home admission within 1 year following cardiac surgery in patients aged 80 years or older. ${ }^{12}$ The difference likely owes to multitude of factors, including the differences in the national health care structures, patient risk profiles, and cultural threshold to nonhome discharge, and may warrant characterization at the national level in the United States.

Predictors of nonhome disposition and 2-year survival following valve surgery in elderly patients were evaluated in a smaller study involving 307 patients, which reported superior 2-year survival in patients discharged to home 

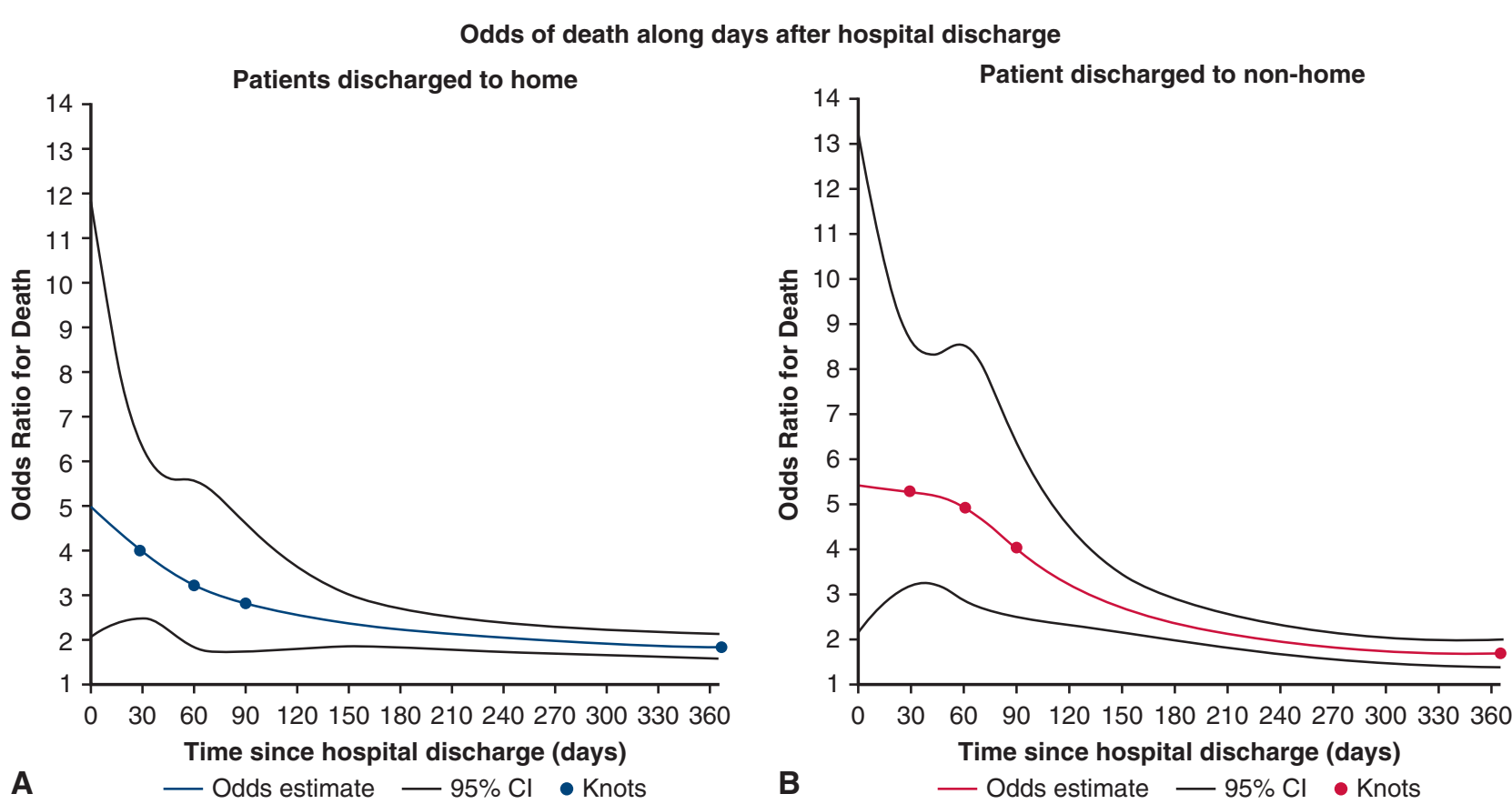

FIGURE 2. Restricted cubic splining for the risk of death along days following hospital discharge. Results of restricted cubic splining demonstrating odds of death along days since the time of discharge. A, Home discharge cohort. B, Nonhome discharge cohort. Solid thick line indicates the odds estimate and thin lines indicate $95 \%$ confidence interval $(C I)$. Knots were defined at 30, 60, 90, and 365 days.

( $80 \%$ vs $90 \% ; P=.01)$. The predictors for nonhome discharge were similar to the ones identified in our study, including female gender, higher number of comorbidity, and the presence of complications. ${ }^{6}$

Both Kaplan-Meier survival estimate and restricted cubic splining indicate that a significant proportion of early posthospital mortality occurs in the nonhospital setting following the index hospital discharge. Although our study design neither intended nor allowed for determining whether it is the nonhome discharge itself that resulted in poor outcomes, our model suggests that non-home discharge is at least a composite marker of adverse events aggregating measured and unmeasured risk factors.

A question raised from the observation of persistently elevated risk of death following hospital discharge is whether prolonging the hospital stay instead of transferring the patient to external facilities would reduce the risk of death. Hospital setting provides more frequent nursing care and rigorous monitoring of the patient's vitals and blood chemistry, although whether such factors result in prevention of adverse event is unknown. The question is pertinent in the current paradigm of value-based purchasing $^{14}$ and restructuring of health delivery systems to decrease hospital costs, of which a common target is the reduction of hospital length of stay. ${ }^{15}$ However, observational studies suggest that early hospital discharge does not appear to result in early return to home because patients stay longer in postacute care facilities before arriving home. ${ }^{16}$
In an alternative scenario in which nonhome discharge itself causes adverse outcomes, hospital-at-home may serve as an effective alternative destination. Hospital-at-home, an emerging paradigm of posthospital care model in which acute hospital-level care is provided at home instead of traditional inpatient rehabilitation care model is associated with reduced risk of readmission and better patient experience. ${ }^{17}$ Such association is untested in surgical populations and may present an opportunity to improve postoperative outcomes. In addition, it is imperative to systematically evaluate the quality of care in the postacute-care phase to identify whether certain centers' care structures may be associated with suboptimal outcomes, which may allow for interventions to improve postacute-phase care.

\section{Limitations}

This study harbors limitation inherently associated with the retrospective and single-center nature limited to the residents of Connecticut State. The completeness of follow-up decreased over time, which was associated with the availability of follow-up data to the end of year 2016. Our analysis indicates that the study was sufficiently powered to detect differences of interest. Although an interpretation of our multivariate model allows for an inference that nonhome discharge was an independent predictor, this does not allow us to conclude whether nonhome discharge itself was the risk factor, or whether nonhome discharge was simply a marker of impending adverse event without any mechanistic involvement. Stroke was defined according 
to the STS Adult Cardiac Surgery Database definition. Therefore, radiographic confirmation of the diagnosis was not required.

\section{CONCLUSIONS}

Hospital discharge to destinations other than home following cardiac surgery was associated with an increased risk of long-term mortality. The postoperative high-risk phase persisted up to 80 days following hospital discharge in patients discharged to locations other than home, whereas this phase was nonexistent in patients discharged to home. This may call for an evaluation of postacute-care quality. Predictors of nonhome discharge were identified, and they may aid in guiding optimization of the clinical status of a patient when possible.

\section{Webcast}

You can watch a Webcast of this AATS meeting presentation by going to: https://aats.blob.core.windows.net/ media/19\%20AM/Monday_May6/202AC/202AC/S86\%20\%20Patient\%20safety/S86_8.mp4 3.

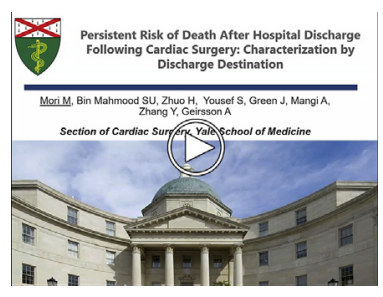

\section{Conflict of Interest Statement}

Authors have nothing to disclose with regard to commercial support.

\section{References}

1. Edwards MB, Taylor KM. Is 30-day mortality an adequate outcome statistic for patients considering heart valve replacement? Ann Thorac Surg. 2003;76:482-5.

2. Siregar S, Groenwold RH, de Mol BA, Speekenbrink RG, Versteegh MI, Brandon Bravo Bruinsma GJ, et al. Evaluation of cardiac surgery mortality rates: 30-day mortality or longer follow-up? Eur J Cardiothorac Surg. 2013;44:875-83.

3. Brooke BS, Goodney PP, Kraiss LW, Gottlieb DJ, Samore MH, Finlayson SR Readmission destination and risk of mortality after major surgery: an observational cohort study. Lancet. 2015;386:884-95.

4. Guduguntla V, Syrjamaki JD, Ellimoottil C, Miller DC, Prager RL, Norton EC, et al. Drivers of payment variation in 90-day coronary artery bypass grafting episodes. JAMA Surg. 2018;153:14-9.

5. Edgerton JR, Herbert MA, Mahoney C, Armstrong D, Dewey TM, Holper E, et al. Long-term fate of patients discharged to extended care facilities after cardiovascular surgery. Ann Thorac Surg. 2013;96:871-7.

6. Henry L, Halpin L, Hunt S, Holmes SD, Ad N. Patient disposition and long-term outcomes after valve surgery in octogenarians. Ann Thorac Surg. 2012;94: 744-50.

7. Levey AS, Bosch JP, Lewis JB, Greene T, Rogers N, Roth D. A more accurate method to estimate glomerular filtration rate from serum creatinine: a new prediction equation. Modification of Diet in Renal Disease Study Group. Ann Intern Med. 1999;130:461-70.

8. Shahian DM, O'Brien SM, Filardo G, Ferraris VA, Haan CK, Rich JB, et al. The Society of Thoracic Surgeons 2008 cardiac surgery risk models: part 1 -coronary artery bypass grafting surgery. Ann Thorac Surg. 2009;88(Suppl):S2-22.
9. Pearce N. What does the odds ratio estimate in a case-control study? Int J Epidemiol. 1993:22:1189-92.

10. Desquilbet L, Mariotti F. Dose-response analyses using restricted cubic spline functions in public health research. Stat Med. 2010;29:1037-57.

11. Shahian DM, O'Brien SM, Sheng S, Grover FL, Mayer JE, Jacobs JP, et al. Predictors of long-term survival after coronary artery bypass grafting surgery: results from the Society of Thoracic Surgeons Adult Cardiac Surgery Database (the ASCERT study). Circulation. 2012;125:1491-500.

12. Thorsteinsson K, Andreasen JJ, Mortensen RN, Kragholm K, Torp-Pedersen C, Gislason G, et al. Longevity and admission to nursing home according to age after isolated coronary artery bypass surgery: a nationwide cohort study. Interact Cardiovasc Thorac Surg. 2016;22:792-8.

13. Ivanov J, Weisel RD, David TE, Naylor CD. Fifteen-year trends in risk severity and operative mortality in elderly patients undergoing coronary artery bypass graft surgery. Circulation. 1998;97:673-80.

14. VanLare JM, Conway PH. Value-based purchasing — national programs to move from volume to value. $N$ Engl J Med. 2012;367:292-5.

15. Leitman IM, Levin R, Lipp MJ, Sivaprasad L, Karalakulasingam CJ, Bernard DS, et al. Quality and financial outcomes from gainsharing for inpatient admissions: a three-year experience. J Hosp Med. 2010;5:501-7.

16. Lazar HL, Fitzgerald CA, Ahmad T, Bao Y, Colton T, Shapira OM, et al. Early discharge after coronary artery bypass graft surgery: are patients really going home earlier? J Thorac Cardiovasc Surg. 2001;121:943-50.

17. Federman AD, Soones T, DeCherrie LV, Leff B, Siu AL. Association of a bundled hospital-at-home and 30-day postacute transitional care program with clinical outcomes and patient experiences. JAMA Intern Med. 2018;178:1033-40.

Key Words: postacute phase, outcome measures, discharge destinations

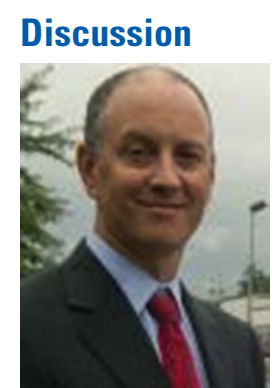

Dr Daniel T. Engelman (Springfield, Mass). I would like to congratulate the authors for an excellent presentation and providing the manuscript for review. A couple of questions come to mind. First of all, from our own data we have noticed that patients who go home do better, but of course those are the healthier patients, so that sort of seems obvious. The sickest patients are the ones that you are sending to a skilled nursing facility and maybe those are the ones more likely to be readmitted or not do as well.

I really have to congratulate you on addressing outcomes beyond 30 days, because cardiac surgeons really have not been focused on that 30-day or 90-day metric. You looked at up to 5 years of follow-up, which is great, because we often focus our research on outcomes analysis over a much shorter time frame.

I also congratulate you on looking at this important understudied area. Historically, anyone older than age 70 to 75 years was going to an SNF-guaranteed-and now we are becoming more selective and realizing that there are consequences to this decision of home versus SNF.

My questions are as follows: Would an extra day or 2 
in the hospital potentially have gotten some patients to go home versus to an SNF.

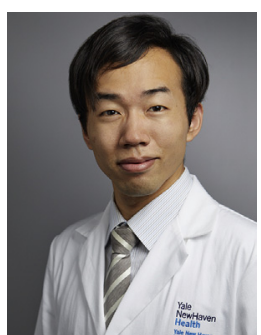

Dr Mori (New Haven, Conn). Thank you for the kind words. That is a great question and we wondered about that as well. Unfortunately, it is not possible to investigate whether longer hospital stay would result in a better outcome retrospectively, because there is inherent bias in the data.

Dr Engelman. What is your personal feeling?

Dr Mori. It may be. However, we believe just converting the discharge location status alone may not be impactful. Discharge destinations are driven by patient factors and complications that primarily influenced the outcome, not necessarily the status of the discharge alone, although that is how we operationalized this analysis.

Dr Engelman. What happens to these patients at 80 days? That's really when they fall off the curve. We talk about falling off the track. Well, something at 80 days happens to these patients in the SNF that made them start to die.

Dr Mori. Actually, the risks persisted for up to 80 days and then after 80 days the risks started to mitigate then normalize.
The question is really what is happening during those 80 days, and we surmised that it is the perioperative hazards that carried over to the time point way beyond 30 days.

Dr Engelman. But only in the SNF group?

Dr Mori. Only in the SNF group.

Dr Engelman. They are okay for 80 days. Initially is when they are dying, then they are okay. So if they make it to 80 days they are all right in the SNF. Aren't most patients sent to SNFs there for 2 or 3 weeks?

Dr Mori. We were not able to investigate that, but anecdotally, yes, about several weeks. I think that would be a great argument against the notion that location of discharge alone confers the risk. Rather, the prolonged risk is likely multifactorial, for reasons such as comorbidity and complications for which those patients had to be discharged to an SNF in the first place.

Dr Engelman. Let's open the floor to other questions regarding SNF versus home. It's a hot topic, and clearly when we had a bundle that rewarded hospitals for sending patients home versus to an SNF, it was a lot bigger emphasis. Now that we are sort of entering an accountable care organization model, the same story: If you really want to save money in an accountable care organization, you get patients to go home at all costs. 

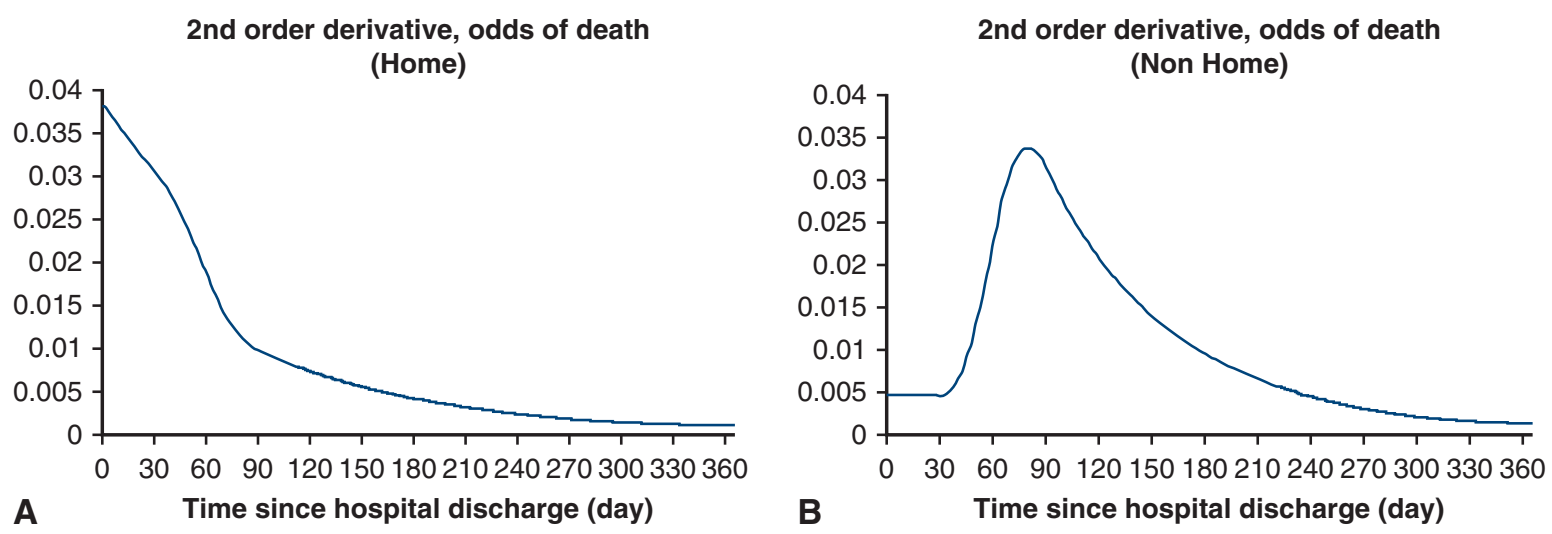

FIGURE E1. Second order derivatives of restricted cubic splining curves. A, Second order derivative of the odds ratio for the risk of death in patients who were discharged to home. There is no transition point in the risk of death. B, Second order derivative of the odds ratio for the risk of death in patients who were discharged to locations other than home. The transition point in the risk of death is the peak at 80 days.

TABLE E1. List of input variables by phase of patient care

\begin{tabular}{|c|c|c|}
\hline Preoperative & Case type & Postoperative \\
\hline Age & Isolated coronary artery bypass graft & Bleeding requiring reoperation \\
\hline Gender & Isolated single valve & Stroke \\
\hline Race & Isolated multiple valve & Prolonged ventilation \\
\hline Body mass index & Coronary artery bypass graft + single valve & Pneumonia \\
\hline Hypertension & Coronary artery bypass graft + multiple valve & Renal failure \\
\hline Diabetes & & Renal failure requiring dialysis \\
\hline Estimated glomerular filtration rate & & Intensive care unit length of stay \\
\hline \multicolumn{3}{|l|}{ Infective endocarditis } \\
\hline \multicolumn{3}{|l|}{ Dialysis } \\
\hline \multicolumn{3}{|l|}{ Lung disease } \\
\hline \multicolumn{3}{|l|}{ Immunosuppressed } \\
\hline \multicolumn{3}{|l|}{ Stroke } \\
\hline \multicolumn{3}{|l|}{ Peripheral vascular disease } \\
\hline \multicolumn{3}{|l|}{ History of percutaneous coronary intervention } \\
\hline \multicolumn{3}{|l|}{ Congestive heart failure } \\
\hline \multicolumn{3}{|l|}{ Case status } \\
\hline \multicolumn{3}{|l|}{ Ejection fraction } \\
\hline \multicolumn{3}{|l|}{ Cardiogenic shock } \\
\hline Redo sternotomy & & \\
\hline
\end{tabular}

